\title{
$\beta$-arrestin protects neurons by mediating endogenous opioid arrest of inflammatory microglia
}

\author{
X Feng ${ }^{1}, \mathrm{C}-\mathrm{Y}$ Wu${ }^{1}$, FH Burton ${ }^{1}, \mathrm{HH}$ Loh $^{1}$ and L-N Wei ${ }^{*, 1}$
}

Microglial activation worsens neuronal loss and contributes to progressive neurological diseases like Parkinson's disease (PD). This inflammatory progression is countered by dynorphin (Dyn), the endogenous ligand of the kappa-opioid receptor (KOR). We show that microglial $\beta$-arrestin mediates the ability of Dyn/KOR to limit endotoxin-elicited production of pro-inflammatory effectors and cytokines, subsequently protecting neurons from inflammation-induced neurotoxicity. Agonist-activated KOR enhances the interaction of $\beta$-arrestin2 with transforming growth factor-beta-activated kinase 1 (TAK1)-binding protein 1 (TAB1), disrupting TAK1-TAB1 mediated pro-inflammatory gene expression. We reveal a new physiological role for $\beta$-arrestin in neuroprotection via receptor internalization-triggered blockade of signal effectors of microglial inflammatory neurotoxicity. This result offers novel drug targets in the convergent $\mathrm{KOR} / \beta$-arrestin2 and inflammatory pathways for treating microglial inflammatory neuropathologies like PD.

Cell Death and Differentiation (2014) 21, 397-406; doi:10.1038/cdd.2013.152; published online 25 October 2013

Increasing evidence suggests that the overactivation of immune system-derived microglia in the substantia nigra (SN) is a key causative factor in the pathogenesis of Parkinson's disease (PD), the most prevalent neurodegenerative disease in the population over 60 years old. Pathologically, PD is characterized by the degeneration of dopaminergic (DA) neurons of the SN pars compacta (SNpc) in the midbrain, leading to disabled voluntary movements. Immunohistochemistry research has indicated that activated microglia accumulate around degenerating neurons in the SN of patients with PD and related parkinsonian syndromes. ${ }^{1}$ Although microglial activation in these diseases is not limited to the SN, the DA neurons in the SN are particularly vulnerable to inflammatory insult owing to the larger population of microglia in the SN. ${ }^{2,3}$ As a sensor of brain injury and aging, microglia's state and activity are regulated through neuronmicroglia communication involving the stimulation of signal transducing or phagocytic microglial receptors by neurotransmitters. ${ }^{4,5}$ Deficiencies in this communication between neurons and microglia as a result of stress or aging leads to reactive microglia and prolonged inflammation and, as a result, neuronal death. Similarly, stimulation of microglia by bacteria or endotoxins results in outbursts of pro-inflammatory cytokines such as interleukin 1 beta (IL-1 $\beta)$, tumor necrosis factor- $\alpha$ (TNF)- $\alpha$, and interleukin 6 (IL-6) through activation of toll-like receptor 4 (TLR4). This can contribute to the death of neurons in the SN because long-term inhibition of IL-1 $\beta$ and TNF- $\alpha$ has consistently been reported to attenuate tyrosine hydroxylase $\left(\mathrm{TH}^{+}\right)$neuron loss in PD models. ${ }^{6,7}$ Meanwhile, neuron degeneration itself leads to further secondary activation of microglia via increased production of matrix metalloproteinase 3 and neuromelaine and, as a result, further neuronal death. ${ }^{8-10}$ Thus, the activation of microglia by an intracerebral injection of an endotoxin such as lipopolysaccharide (LPS) is sufficient to cause the loss of $\mathrm{TH}^{+}$ neurons. ${ }^{11}$ However, it has remained elusive how microglial activity is regulated.

The kappa-opioid receptor (KOR), which belongs to the $\mathrm{Gi}$ (inhibitory) protein-coupled seven transmembrane receptor (GPCR) superfamily, is one of the three major subtypes of receptors mediating the effects of opioid neurotransmitters. Functionally, KOR is activated by its endogenous agonist, dynorphin (Dyn), which is abundantly released in the $\mathrm{SN}$, as well as by exogenous ligands. ${ }^{12}$ Agonist binding to KOR causes the receptor to be internalized, through the formation of a KOR/ $\beta$-arrestin2 complex, in the G-protein-coupled receptor kinase 3 phosphorylation-dependent GPCR desensitization pathway. Such internalization/desensitization prevents further activation of KOR, thereby reducing receptor signaling; however, other GPCR/ $\beta$-arrestin2 complexes have more recently been shown to regulate mitogen-activated protein kinase and other intracellular signal transduction pathways. ${ }^{13} \mathrm{KOR}$ is predominantly expressed in neurons, and particularly so in DA neurons of the central nervous system where it modulates the release of glutamate, acetylcholine, and dopamine. This supports KOR's profound effects on both brain's reward and locomotor systems. ${ }^{14}$ For example, the specific KOR agonist TRK-820 reduces

\footnotetext{
${ }^{1}$ Department of Pharmacology, University of Minnesota Medical School, Minneapolis, MN 55455, USA

*Corresponding author: L-N Wei, Department of Pharmacology, University of Minnesota Medical School, 6-120 Jackson Hall, 321 Church Street SE, Minneapolis, MN 55455, USA. Tel: +612 6259 402; Fax: +612 6258 408; E-mail: weixx009@umn.edu

Keywords: $\beta$-arrestin; microglial inflammation; neurotoxicity; dynorphin; kappa-opioid receptor

Abbreviations: CM, conditioned medium; DA, dopaminergic; Dyn, dynorphin; GPCR, Gi (inhibitory) protein-coupled seven transmembrane receptor; IKK, I $\kappa$-B kinase; IL-1 $\beta$, interleukin 1 beta; IL-6, interleukin 6; iNOS, inducible nitric oxide synthase; KOR, kappa-opioid receptor; LPS, lipopolysaccharide; NF- $\kappa$ B, nuclear factor-kappa B; NorB, norbinaltorphimine; PD, Parkinson's disease; PLA, proximal ligation assay; SN, substantia nigra; SNpc, SN pars compacta; TAB1, TAK1-binding protein 1; TAK1, transforming growth factor-beta-activated kinase 1; TH, tyrosine hydroxylase; TLR4, toll-like receptor 4; TNF- $\alpha$, tumor necrosis factor- $\alpha$

Received 12.4.13; revised 12.8.13; accepted 13.9.13; Edited by L Greene; published online 25.10.13
} 
L-dopa-derived extracellular dopamine content, and as a result ameliorates dopamine replacement drug-overdose dyskinesia, in the striatal 6-hydroxydopamine-lesioned rat PD model, although not worsening their basal level of parkinsonism. ${ }^{15}$ Neurotransmitter release studies have similarly revealed that synthetic KOR agonists $\mathrm{U} 50,488 \mathrm{H}$ and U69,593 decrease activity-stimulated dopamine release from DA neurons but have no effect on their basal activity. ${ }^{16,17}$ Moreover, the mRNA level of Dyn was reduced in the SN in postmortem brain specimens of both $\mathrm{PD}$ patients and an animal model of PD. ${ }^{18,19}$ These suggest Dyn/KOR has a presynaptic inhibitory role in DA neurotransmission and a modulatory role in progressive PD pathogenesis. Until recently, it was presumed that all such influences by Dyn/KOR were mediated only through neuronal action. However, interestingly, a recent study demonstrated that, in MPTP-lesioned rodent PD models, Dyn deficiency exacerbates DA neuron loss, not as one might have presumed through disinhibitory stress on residual DA terminals, but apparently through the amplified activation of inflammatory microglia. ${ }^{20}$ How Dyn/KOR signaling could regulate microgliamediated DA neuronal loss bears further investigation.

Although KOR's predominant expression is within neurons, there is emerging evidence that KOR is also highly expressed and functional in immune system cells. ${ }^{21,22}$ For example, the KOR agonist U50,488H blocks LPS-induced production of IL-1 $\beta$, IL-6, and TNF- $\alpha$ in the macrophage cell line P388D1, an effect reversed by both opioid antagonist naloxone and the KORspecific antagonist norbinaltorphimine (NorB). ${ }^{23}$ However, $\mathrm{U} 50,488 \mathrm{H}$ failed to modulate the production of pro-inflammatory cytokines in a macrophage-derived cell line, RAW 264.7, ${ }^{24}$ suggesting that different subpopulations of macrophages exist with different sensitivities to KOR agonists. Microglia are immune cells and express KOR. ${ }^{25}$ Studies show KOR activation exerts immune protection: KOR agonists inhibit human immunodeficiency virus 1-mediated neurotoxicity in acutely infected human microglia; ${ }^{25,26}$ and selective KOR agonists attenuate the production of inflammation-mediating superoxide anions from activated human fetal-derived microglia. ${ }^{27}$

Because of the pivotal role microglial inflammatory activity has in neuron loss such as in PD pathogenesis, it is critical to know what intracellular signal pathway in microglia regulates this inflammatory process, and how anti-inflammatory signals like Dyn/KOR may regulate this process. Here we reveal microglial $\beta$-arrestin to be a critical anti-inflammatory signal mediator. Its signal input, microglial Dyn/KOR, activates $\beta$-arrestin to sequester pro-inflammatory TAB1 (transforming growth factor-beta-activated kinase 1 (TAK1)-binding protein 1) away from TAB1's molecular conspirators, thereby arresting the downstream inflammatory pathway. In contrast, the loss of microglial KOR or $\beta$-arrestin activity enhances pro-inflammatory signaling, prolongs SN microglial reactivity, and aggravates SN DA neuron loss in an endotoxin-induced PD model.

\section{Results}

Microglial KOR protects $\mathrm{TH}^{+}$-neurons from inflammation-induced cell death. KOR activation in DA neuronal cells affects the state of the brain reward system and behavior by regulating neuronal activity. ${ }^{28}$ However, the high level of KOR in adjacent non-neuronal cells, particularly in immune-derived microglia such as those in the SN associated with neurodegeneration in PD, has raised the question of whether it has a regulatory role in inflammatory microglial exacerbation of neurodegenerative disorders. To define what cell types are responsible for endotoxin-mediated neurotoxicity in wild-type (WT) versus KOR knockout $\left(\mathrm{Kor}^{-/-}\right)$ animals, we explored the in vitro effects of LPS-treated microglia and astrocytes derived from WT or $\mathrm{Kor}^{-/-}$mice on $\mathrm{SN}$-derived $\mathrm{TH}^{+}$neurons from both WT and $\mathrm{Kor}^{-/-}$mice. Figure 1a shows that the incubation of neurons with a conditioned medium (CM) from LPS-treated $\mathrm{Kor}^{-/-}$microglia resulted in a nearly $40 \%$ greater decrease of neuron viability than with such CM from WT microglia. Interestingly, no difference in cell viability was detected between the WT and $\mathrm{Kor}^{-/-}$neurons after treatment with WT or $\mathrm{Kor}^{-/-}$ microglia-derived $\mathrm{CM}$, indicating that microglia, not neurons, are involved in the aggravation of endotoxin-induced neurotoxicity in $\mathrm{Kor}^{-/-}$animals.

A previous study reported that microglia-initiated neurotoxicity is amplified by astrocytes. ${ }^{29}$ To further define KOR's potential role in astrocytes to mediate inflammation-induced neurotoxicity, we assayed the survival of $\mathrm{TH}^{+}$neurons from WT mice after incubating the neurons with CM from LPStreated cultures of either astrocytes alone, microglia alone, or microglia-astrocyte mixture, from either WT or $\mathrm{Kor}^{-/-}$mice. The results (Figure 1b) indicate that the CM from LPS-treated $\mathrm{Kor}^{-/-}$microglia or a microglia-astrocyte mixture with $\mathrm{Kor}^{-1}$ microglia (M-Kor ${ }^{-/}$), but interestingly not from astrocytes alone or a microglia-astrocyte mixture with $\mathrm{Kor}^{-/-}$astrocyte $\left(\mathrm{A}-\mathrm{Kor}^{-/-}\right)$, decreased neuronal viability relative to the $\mathrm{CM}$ from their WT counterpart LPS-treated cells. These results demonstrate that KOR deficiency in astrocytes is not responsible for the aggravation of endotoxin-induced neurotoxicity.

To further define the neuronal protective potential of KOR in microglia after exposure to endotoxins, $\mathrm{TH}^{+}$neurons were directly cocultured with WT or $\mathrm{Kor}^{-/-}$microglia that had been LPS- or treated with LPS plus Dyn (LPS + Dyn), Dyn being an endogenous agonist of KOR protein. Consistent with the $\mathrm{CM}$ studies, Figure 1c shows that after a 24-h coculture of neurons with LPS-treated WT or $\mathrm{Kor}^{-/-}$microglia, there was a greater decrease in the number of $\mathrm{TH}^{+}$cells in neurons cocultured with $\mathrm{Kor}^{-/-}$microglia. Similarly, Dyn co-treatment could only reverse the decrease of $\mathrm{TH}^{+}$cells cocultured with WT microglia, not with $\mathrm{Kor}^{-1-}$ microglia. Further, pathological morphology of $\mathrm{TH}^{+}$neurons - reduced or absent processes and alterations in cell size and shape - was more prevalent in the neurons cocultured with $\mathrm{Kor}^{-/-}$microglia than with WT microglia (Supplementary Figure S1c). Similarly, this coculture of $\mathrm{TH}^{+}$neurons with LPS-treated $\mathrm{Kor}^{-/-}$microglia also resulted in increased apoptotic cells indicated by TUNEL and pSIVA-PI staining (Figure 1d and Supplementary Figure S1d). Thus, KOR in microglia has a critical and direct role in protecting neurons from endotoxin-induced neurotoxicity.

Agonist-activated microglial KOR inhibits endotoxininduced neurotoxic factor production. On the basis of described results that KOR in microglia protects neurons from endotoxin-induced neurotoxicity, and given that 

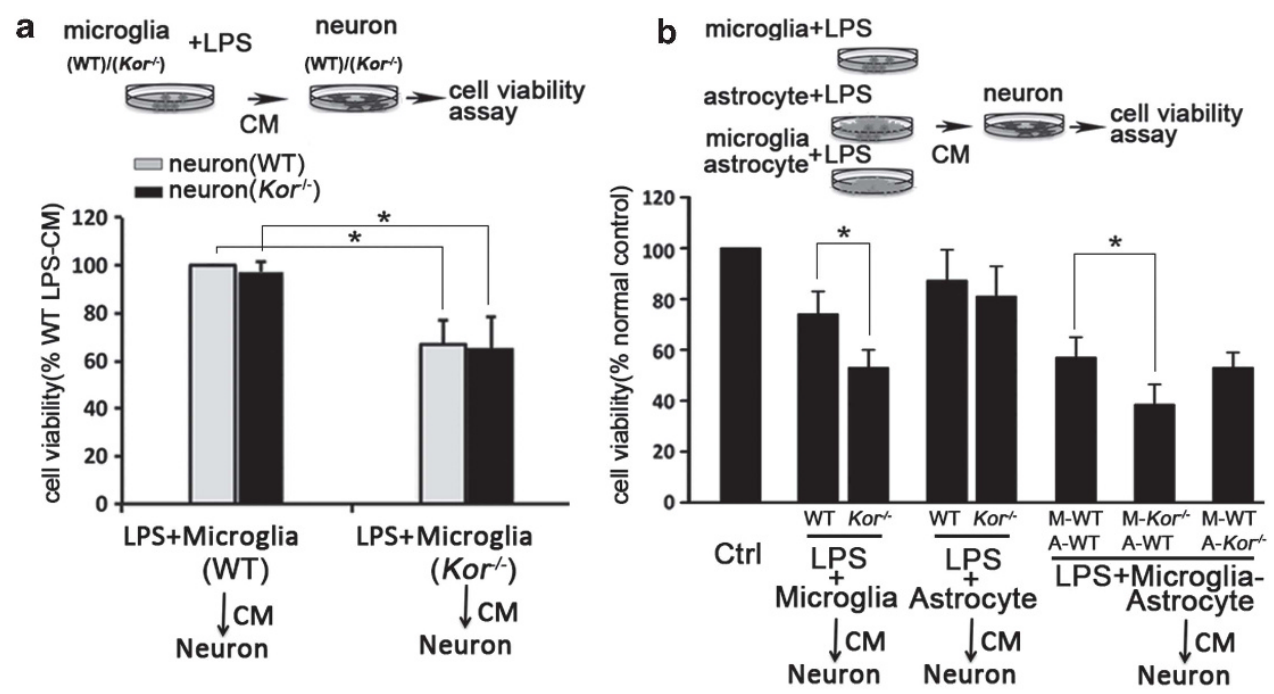

C
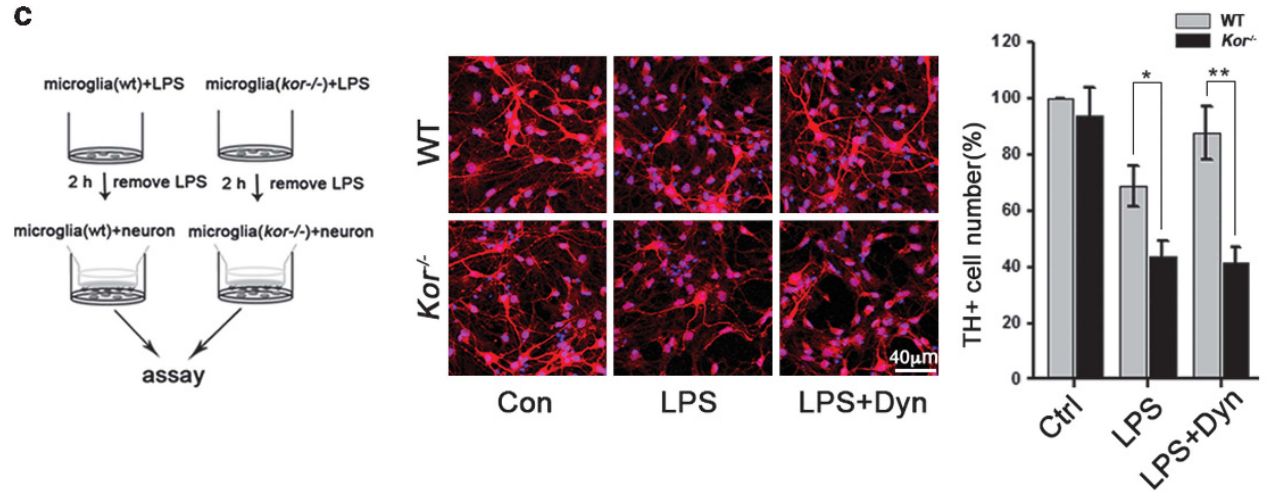

d
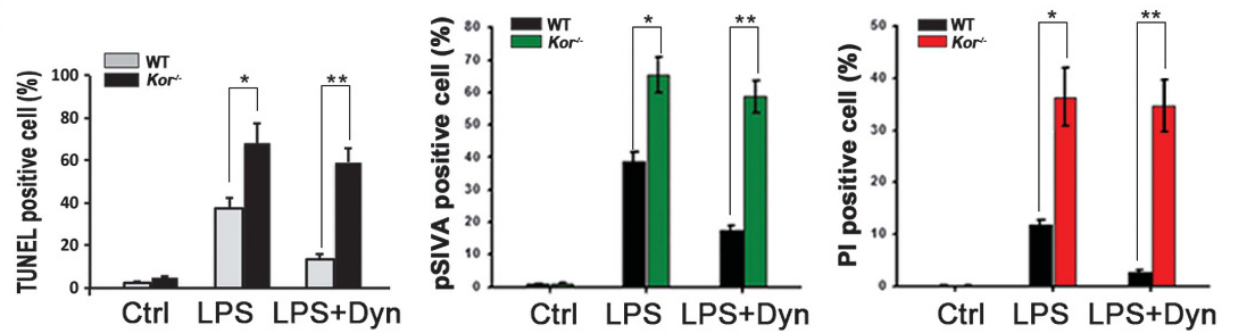

Figure 1 Microglial KOR protects $\mathrm{TH}^{+}$neurons from inflammatory neurotoxicity. (a) Cell viability assay of DA neurons from wild-type (WT) or KOR knockout (Kor ${ }^{-/-}$) mice, cultured with condition medium (CM) from LPS-treated microglia from WT (CM-WT) or $\mathrm{Kor}^{-/-}\left(\mathrm{CM}-\mathrm{Kor}^{-/-}\right)$mice. (b) Cell viability assay of DA neurons cultured $24 \mathrm{~h}$ with CM from LPS-treated microglia and astrocytes from WT or Kor ${ }^{-1-}$ mice, WT microglia (M-WT)-WT astrocyte (A-WT) mixture, Kor $^{-1-}$ microglia (M-Kor ${ }^{-1-}$ )-WT astrocyte (A-WT) and WT microglia (M-WT)-Kor ${ }^{-1-}$ astrocyte (A-Kor ${ }^{-1-}$ ) mixture. The neuron cultured with normal medium was normal control (Ctrl). (c) Image of $\mathrm{TH}^{+}$neurons cocultured with microglia (WT or $\mathrm{Kor}^{-1-}$ ) following LPS-only or LPS + Dyn treatment. Quantification of $\mathrm{TH}^{+}$cell number is indicated in right graphic panel. (d) DA neurons from WT mice were cocultured with microglia (WT or Kor ${ }^{-1-}$ ) treated with LPS-only or LPS + Dyn for $2 \mathrm{~h}$. After $24 \mathrm{~h}$ coculture, the apoptotic neurons were indicated by TUNEL-positive staining or pSIVA-PI staining. Values are the means \pm S.D. in separate experiments $(n=3)$ with significance ${ }^{*} P<0.05,{ }^{* *} P<0.01$ versus respective WT group (Student's $t$-test)

endotoxin-induced neurotoxicity is mainly mediated by the increased production of pro-inflammatory cytokines, ${ }^{30}$ we then determined how KOR affects microglial cytokine activity in response to LPS treatment. Figure $2 \mathrm{a}$ shows that the gene expression level of inflammatory mediators $I L-1 \beta, T N F-\alpha$, and inducible nitric oxide synthase (iNOS) was significantly increased in $\mathrm{Kor}^{-1-}$ microglia compared with WT microglia after LPS treatment. Dyn was able to reduce the LPSinduced increase of the inflammatory mediators in WT microglia; however, Dyn lost this ability in $\mathrm{Kor}^{-/-}$microglia.
Dyn's ability to inhibit the LPS-induced increase of TNF- $\alpha$ protein was similarly abolished in murine microglial BV2 cells upon transfection with KOR siRNA (Supplementary Figure $\mathrm{S} 2 \mathrm{~b})$. Moreover, the administration of NorB, a specific KOR antagonist, rendered Dyn unable to reduce the LPS-induced increase of IL-1 $\beta$ and TNF- $\alpha$ protein levels in WT mouse microglia (Figure 2b). We also found that, like Dyn, many synthetic, exogenous KOR agonists were able to inhibit the LPS-induced increase of inflammatory mediators (Supplementary Figure S2a). In addition to altering the gene 

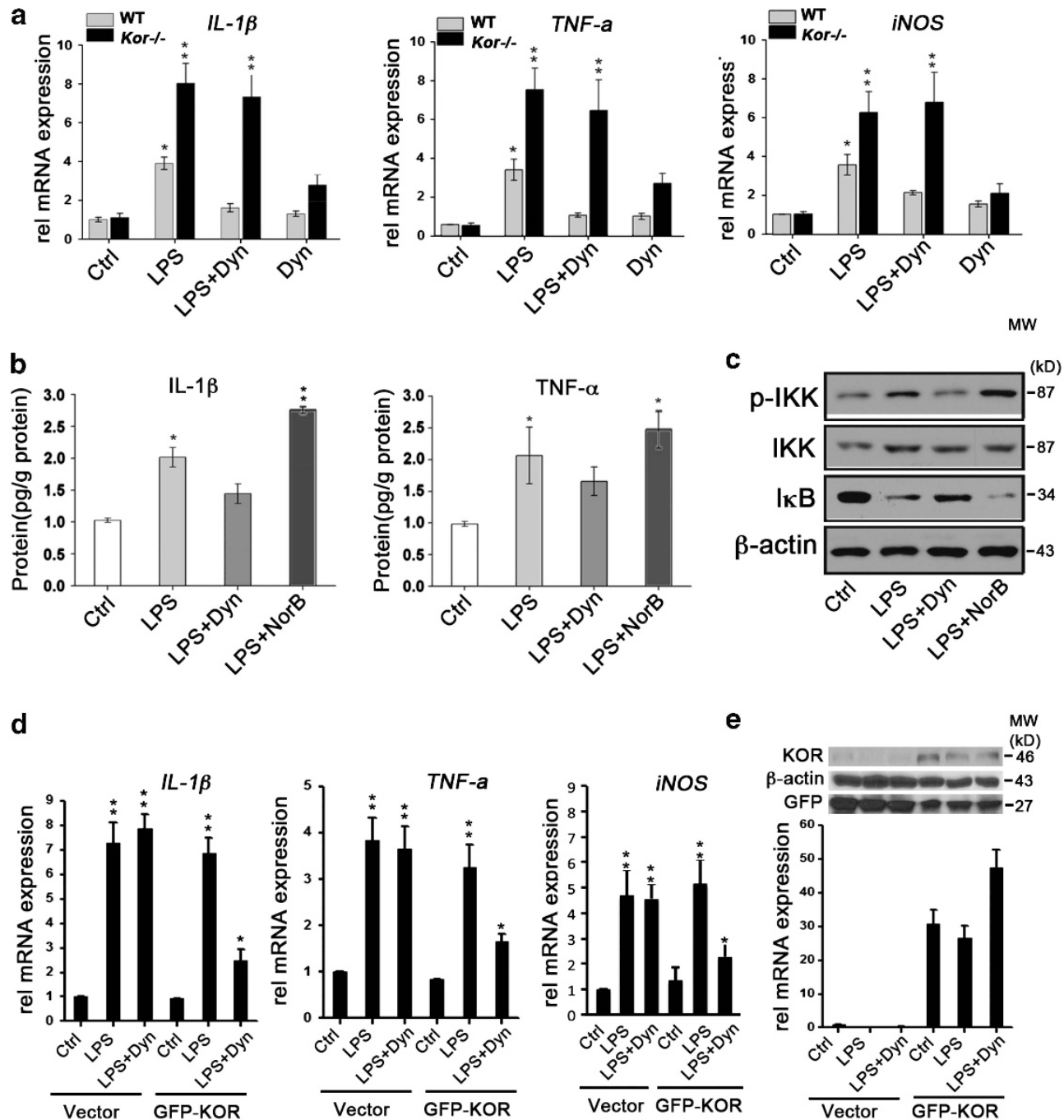

Figure 2 Microglial KOR inhibits production of neurotoxic factors. (a) Expression level of IL-1 $\beta, T N F-\alpha$ and iNOS transcripts detected by qPCR in WT or Kor ${ }^{-/-}$microglia after $2 \mathrm{~h} \mathrm{LPS}$, LPS + Dyn, or Dyn treatment. (b) Expression level of TNF- $\alpha$ and IL-1 $\beta$ protein detected by ELISA in WT microglia after $2 \mathrm{~h}$ treatment with LPS $+/-$ Dyn (KOR agonist) or NorB, a KOR antagonist. (c) Effects of prior KOR agonist/antagonist on LPS-induced IKK phosphorylation and IKB degradation is indicated by western blotting in WT microglia; $\beta$-actin was the loading control. (d) Expression level of IL-1 $\beta$, TNF- $\alpha$ and $i N O S$ mRNA in $\mathrm{Kor}^{-1}$ - microglia transfected with KOR-IRES-GFP vector and then treated with LPS-only or LPS + Dyn for $2 \mathrm{~h}$. (e) KOR protein and mRNA level in $\mathrm{Kor}^{-1-}$ microglia transfected with KOR-IRES-GFP vector; $\beta$-actin and GFP were loading controls. Values are the means \pm S.D. of separate experiments $(n=3)$ with significance ${ }^{*} P<0.05,{ }^{* *} P<0.01$ versus respective control group (Student's $t$-test)

expression of inflammatory mediators, treating microglia with Dyn inhibits the LPS-induced increase of I $\kappa$-B kinase (IKK) phosphorylation and $\mathrm{I}_{\kappa} \mathrm{B}$ degradation, with Dyn inhibition abolished by both NorB treatment (Figure 2c) and, in BV2 cells, transfected KOR siRNA (Supplementary Figure S2c). Further, expressing a KOR-expressing vector (KORIRES-GFP) in $\mathrm{Kor}^{-/-}$microglia restored Dyn's ability to inhibit pro-inflammatory mediator production (Figures $2 \mathrm{~d}$ and e), further demonstrating that the presence and activation of KOR in microglia is essential to inhibit their endotoxininduced inflammatory response.

It is well established that nuclear factor-kappa B (NF- $\kappa$ B) p65 nuclear translocation is necessary to activate TLR4-mediated gene regulation in immune system cells following endotoxin exposure. Supplementary Figure S3a shows that Dyn treatment inhibits the LPS-induced p65 nuclear translocation in WT microglia but not in $\mathrm{Kor}^{-/-}$ microglia. The same result is obtained in microglial BV2 cells by siRNA-based KOR knockdown (Supplementary Figure S3b) or by NorB treatment (Supplementary Figure S3c).

Agonist-activated KOR depends on $\beta$-arrestin2 to elicit anti-inflammatory effects. As the major regulator and adaptor of the GPCR signaling pathway, $\beta$-arrestins have a critical role in the regulation of opioid receptor-mediated function. ${ }^{31,32}$ Interestingly, the expression level of $\beta$-arrestin2 was found to be decreased as a result of TLRs activation, through transcriptional and translational mechanisms, ${ }^{33}$ and in mouse embryonic fibroblast cells $\beta$-arrestin2 has been reported to negatively regulate TLR4-mediated NF- $\kappa \mathrm{B}$ activation. ${ }^{34}$ To investigate the potential involvement of $\beta$-arrestin signaling in KOR-mediated anti-inflammation in microglia, we first checked the expression level of $\beta$-arrestin proteins in BV2 cells at different time points 
after Dyn treatment. Figure 3a shows a robust increase in $\beta$-arrestin2 protein levels as soon as $10 \mathrm{~min}$ following Dyn treatment, whereas $\beta$-arrestin1 remained the same before and after Dyn treatment. Meanwhile, $\beta$-arrestin2 mRNA levels showed no significant increase after Dyn treatment (Supplementary Figure S4). The absence of Dyn-induced transactivation of $\beta$-arrestin2 mRNA, together with the finding in Figure $3 \mathrm{~b}$ that blocking translation, instead of transcription, prevents $\beta$-arrestin2 protein from being increased by Dyn, demonstrated that KOR activation as a result of Dyn stimulation increases the translation efficiency and consequently the level of $\beta$-arrestin2 protein.

We then reduced microglia $\beta$-arrestin2 mRNA with siRNA against $\beta$-arrestin2 (Si $\beta$-arr2; and thus any availability for later translational induction by Dyn). As seen in Figure $3 c$, the reduction of $\beta$-arrestin2 expression in microglia exaggerated the gene expression of inflammatory mediators in response to LPS and, interestingly, abolished the effect of Dyn. It is well known that the increased expression of pro-inflammatory cytokines induced by endotoxins depends on the activation of TLRs signaling pathways, including the activation of TAK1, $\mathrm{IKK}$, and then NF- $\kappa \mathrm{B}$ nuclear translocation upon IkB degradation. ${ }^{35}$ The present study indicates that Dyn can inhibit the activation of the TLR signaling pathway, including upregulated phosphorylation of TAK1 and IKK, and enhanced nuclear translocation of NF- $\kappa \mathrm{B}$, but not in $\beta$-arrestin2 knockdown microglia (Figures $3 \mathrm{~d}$ and $\mathrm{e}$ ). These results demonstrate that the anti-inflammatory function of KOR in microglia depends on $\beta$-arrestin2.

$\beta$-arrestin2 interrupts the TLR4 signaling pathway by interaction with TAB1. As the signal transduction of the inflammatory response through the TLR4 pathway depends on the activation of TAK1 by association of TAK1 with $\mathrm{TAB} 1,{ }^{36}$ we hypothesized that the $\beta$-arrestin2-dependent anti-inflammation function of Dyn/KOR signaling could involve disrupting this TLR4-mediated TAK1-TAB1 association. Thus, we examined the in vivo association of TAB1 and TAK1 with $\beta$-arrestin2 by in situ proximal ligation assay (PLA) and co-immunoprecipitation assays. In Figure 4a, PLA assays showed endogenous TAK1-TAB1 association was significantly increased upon LPS treatment and further enhanced in $\mathrm{Kor}^{-/-}$microglia. Dyn inhibited the association between TAK1 and TAB1 in WT microglia; however, Dyn lost this function in $\mathrm{Kor}^{-/-}$microglia. Notably, an association between TAB1 and $\beta$-arrestin2 was detected in resting
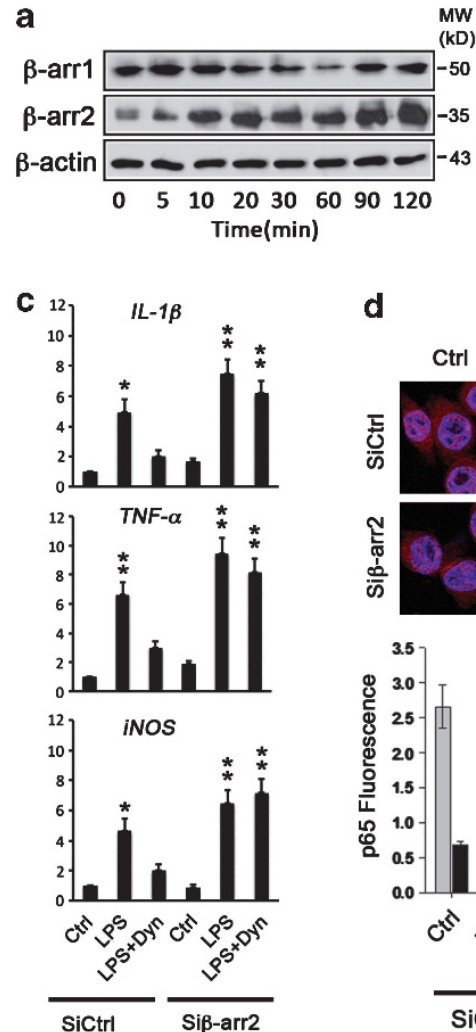

d

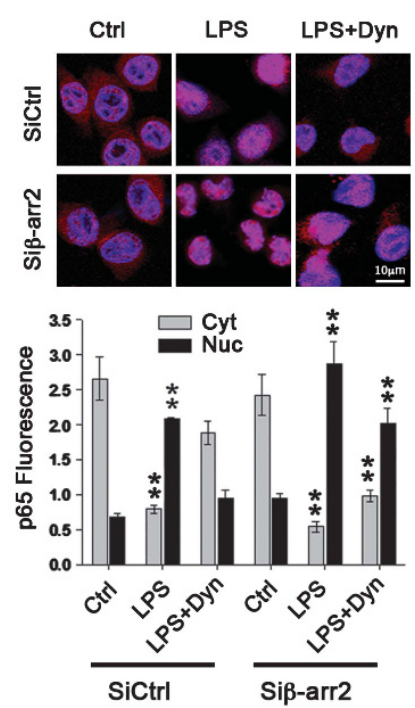

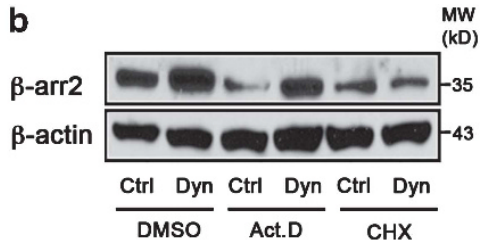

$\mathbf{e}$

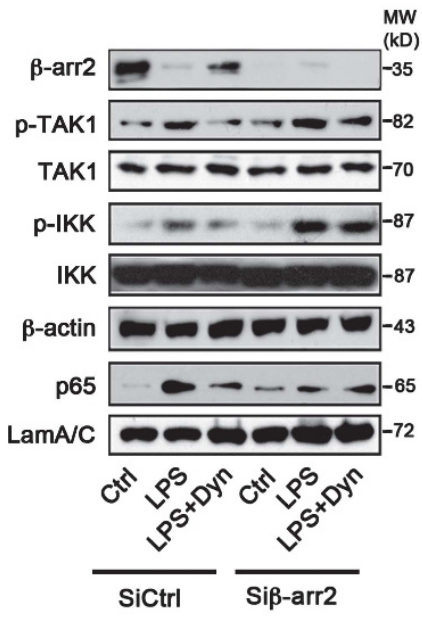

Figure 3 KOR inhibits LPS-induced inflammation via $\beta$-arrestin2. (a) Time course of expression levels of $\beta$-arrestin1 versus $\beta$-arrestin2 in microglial BV2 cells after Dyn treatment. The right graph represents the relative ratio of $\beta$-arrestin1 and $\beta$-arrestin2 protein level to $\beta$-actin. (b) $\beta$-arrestin2 protein level in BV2 cells after Dyn treatment for $2 \mathrm{~h}$ following treatment of cells with transcription inhibitor actinomycin D (Act.D) or translation inhibitor cycloheximide (CHX) for $1 \mathrm{~h}$. (c) Expression of IL-1 $\beta$, TNF- $\alpha$ and $i N O S$ mRNA levels in WT microglia transfected with $\beta$-arrestin2 siRNA (Si $\beta$-arr2) or control vector (SiCntrl), and then treated with LPS-only or LPS + Dyn for $2 \mathrm{~h}$. (d) NF- $k B$ p65 fluorescence image of Si $\beta$-arr2- or SiCntrl-transfected WT microglia treated with LPS-only or LPS + Dyn for $2 \mathrm{~h}$, with lower panel showing quantification of NF- $\kappa$ B p65 fluorescence intensity in the cytoplasm (Cyt) and nucleus (Nuc). (e) Expression of $\beta$-arrestin2 and inflammation signaling transduction-related proteins in Si $\beta$-arr2- or SiCntrltransfected BV2 microglia treated with LPS-only or LPS + Dyn for $2 \mathrm{~h} ; \beta$-actin was the loading control for $\beta$-arrestin2 ( $\beta$-arr2), TAK1 and IKK, whereas laminA/C (LamA/C) was the loading control for nuclear NF- $k B$ p65 (p65). Values are the means \pm S.D. in separate experiments $(n=3)$ with significance ${ }^{*} P<0.05,{ }^{* *} P<0.01$ versus respective control group (Student's $t$-test) 
microglia and decreased in response to LPS treatment. No association of $\beta$-arrestin2 with TAK1 was detected in either resting or LPS-reactive states of microglia. Consistently, Dyn treatment increased the TAB1 and $\beta$-arrestin2 association only in WT but not $\mathrm{Kor}^{-1-}$ microglia (Figure 4b). An enhanced association of TAB1 with $\beta$-arrestin2 and decreased association of TAB1 with TAK1 by Dyn treatment was further confirmed by co-immunoprecipitation in murine microglial BV2 cells (Figure 4c), which likewise confirmed that KOR knockdown limited Dyn's ability to increase the association of TAB1 with $\beta$-arrestin2 or decrease the association of TAB1 with TAK1 in these microglia. These findings confirm that the neuroprotective, anti-inflammatory function of microglial Dyn/KOR receptor stimulation is to disrupt TAB1's pro-inflammatory association with TAK1 by enhancing TAB1's anti-inflammatory association with $\beta$-arrestin2.

KOR-initiated microglial anti-inflammatory signaling
protects $\mathrm{TH}^{+}$-neurons in animals. To confirm KOR-initiated microglial anti-inflammatory signaling has a neuroprotective role in vivo, we stereotaxically injected LPS into the SN of WT or $\mathrm{Kor}^{-1-}$ mice to induce inflammation, a well-established method to study endotoxin aggravation of PD development. LPS + / - Dyn was injected to activate microglia with or without parallel KOR activation. As increased $\mathrm{TH}^{+}$cell death in the SN is the hallmark of PD, we examined the loss of $\mathrm{TH}^{+}$neurons in the SN, 3 weeks after injection. The results showed a predictable decrease in the number of $\mathrm{TH}^{+}$neurons after LPS injection in WT mice but dramatically showed much worse $\mathrm{TH}^{+}$neuron loss in $\mathrm{Kor}^{-/-}$mice. Tellingly, LPS + Dyn injections protected the $\mathrm{TH}^{+}$neurons in the SN of WT mice but failed to do so in $\mathrm{Kor}^{-/-}$mice, showing the microglialmediated neuroprotective function of Dyn is abolished by KOR knockout (Figures $5 \mathrm{a}$ and b and Supplementary Figure S6, Supplementary Table S1). Similarly, TUNEL-positive stained apoptotic cells were increased in both LPS- and LPS + Dyninjected $\mathrm{Kor}^{-/-}$mice (Figure 5c).

We then confirmed by quantitative real-time PCR (qPCR) that the gene expression of the microglial inflammatory cytokines $I L-1 \beta, T N F-\alpha$, and iNOS was increased in the SN of these animals $6 \mathrm{~h}$ after stereotaxic LPS injection (Figure 5d). Again, compared with the WT group, the gene expression of these microglial inflammatory mediators was greatly exaggerated in $\mathrm{Kor}^{-/-}$animals. Dyn co-injection inhibited this increase in WT mice but lost the ability to block the

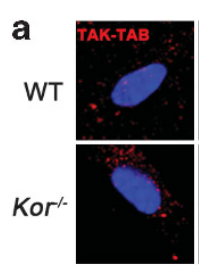

Ctrl

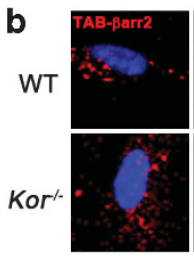

Ctrl

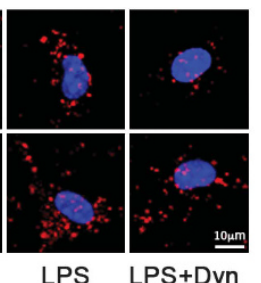

LPS LPS+Dyn

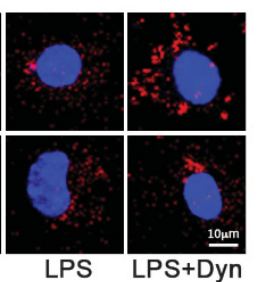

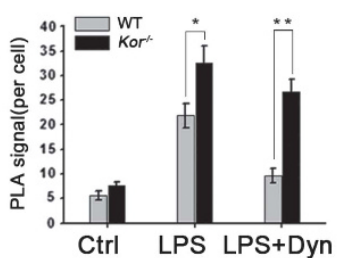

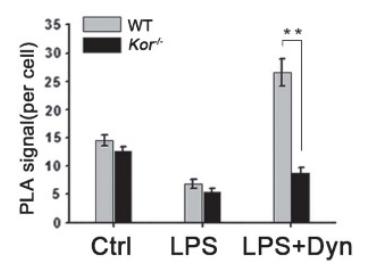

c

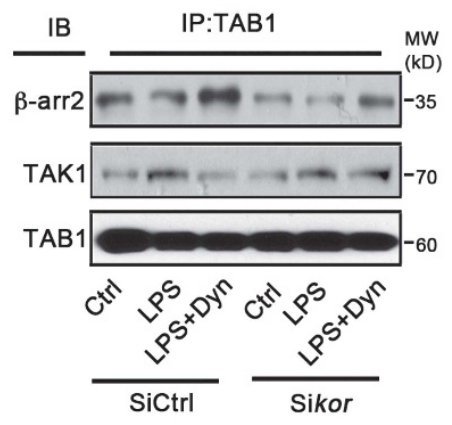

Figure 4 KOR activation enhances TAB1 and $\beta$-arrestin2 interaction. (a) Interaction of TAB1 with TAK1 indicated (as red fluorescent punctae) by in situ PLA assay using antibodies of rabbit anti-TAB1 and mouse anti-TAK1 in WT or $\mathrm{Kor}^{-1-}$ microglia after $2 \mathrm{~h}$ LPS-only or LPS + Dyn treatment. The right graph quantifies (as red fluorescent punctae counts) the interaction of TAB1 with TAK1. (b) Interaction of TAB1 with $\beta$-arrestin2 is indicated (as red punctae) by PLA assay using antibodies of rabbit anti-TAB1 and mouse anti- $\beta$-arrestin2 in microglia from WT or $K^{-1-}$ mice after $2 \mathrm{~h} \mathrm{LPS-only} \mathrm{or} \mathrm{LPS}+$ Dyn treatment. The right graph quantifies (as red fluorescent punctae counts) the interaction of TAB1 with $\beta$-arrestin2. (c) Interaction of TAB1 with $\beta$-arrestin2 and TAK1 is indicated by co-immunoprecipitation assay in microglial BV2 cells transfected with KOR siRNA (Sikor) or control (SiCntrl) vector, and then treated with LPS-only or LPS + Dyn for $2 \mathrm{~h}$. Values are the mean \pm S.D. in separate experiments $(n=3)$ with significance ${ }^{*} P<0.05,{ }^{* *} P<0.01$ versus respective WT group (Student's $t$-test)

Figure 5 Enhanced microglial activation and $\mathrm{TH}^{+}$neuronal lose in $\mathrm{Kor}^{-/-}$mice after LPS administration. LPS or LPS plus dynorphin (LPS + Dyn, $\left.5 \mathrm{nmol}\right)$ diluted in $1 \mu \mathrm{l}$ normal saline (NS) were injected into wild-type (WT) or KOR knockout (Kor ${ }^{-/}$) mice's left SN; the same volume of NS was injected into the right SN as control (Ctrl). Six hours later, three of the animals were killed to test gene expression and the other animals were killed after 21 days for immunohistochemistry. (a) IF staining of $\mathrm{TH}_{\mathrm{TH}} \mathrm{TH}^{+}$cells (red) in mice's brains, with nuclei labeled by DAPI (blue). (b) Quantification of cell survival indicated by $\mathrm{TH}^{+}$cell number obtained from unbiased stereological estimation. The result shows the percentage of $\mathrm{TH}^{+}$cell number from treated groups to the WT control group. (c) Quantitative results of cell apoptosis indicated by TUNEL-positive cell number in SN of different animals after 21 days of injection. (d) Expression level of relative pro-inflammatory cytokines IL-1 $\beta, T N F-\alpha$ and iNOS mRNA by detected by qPCR in SN $6 \mathrm{~h}$ after LPS or LPS + Dyn injection. All statistical values are mean \pm S.D. in separate experiments $(n=3-5)$ with significance ${ }^{*} P<0.05,{ }^{\star \star} P<0.01$ versus respective WT group (Student's t-test). (e) Model of anti-inflammation pathway of KOR/ $\beta$-arrestin2 signaling in microglia. In the SN, Dyn is released by neurons and activates its receptor, KOR, which is expressed in microglia as well as neurons. In contrast, endotoxins such as lipopolysaccharide (LPS) exert more selective action upon microglia. Left: exposure of microglia to an endotoxin-like LPS causes microglial activation through the TLR4 signaling pathway, which triggers inflammatory (M1-phenotype) microglia differentiation and results in DA neuronal death. Activation of KOR in microglia by the receptor's endogenous agonist (Dyn) inhibits LPS-induced activation of downstream TLR4 signaling, by competitive recruitment of $\beta$-arrestin2-TAB1 interaction, reducing microglial overactivation and release of neurotoxic factors, thus protecting DA neurons from inflammationinduced cell death. Right: lack of either KOR (via Kor ${ }^{-1-}$ gene knockout or siRNA knockdown) or $\beta$-arrestin2 (via siRNA knockdown) in microglia disrupts the Dyn/KOR/ $\beta$ arrestin2 anti-inflammatory pathway to deregulate microglial activation, which now becomes superinflammatory in response to endotoxin exposure 
increased production of the pro-inflammatory genes in $\mathrm{Kor}^{-/-}$ mice. Interestingly, the number of microglia, indicated by CD11b-positive staining, was significantly increased $24 \mathrm{~h}$ after LPS administration, and Dyn co-injection reduced this microglial amplification (Supplementary Figure S5), confirming that an essential in vivo neuroprotective role is had by KOR-initiated arrest of microglial inflammatory signaling.

\section{Discussion}

Evidence that inflammation is associated with PD neuropathology includes the increased activation of microglia and of pro-inflammatory mediators in the SNpc of PD patients, ${ }^{3,37}$ and the therapeutic benefit to $P D$ patients of resolving the inflammatory response. ${ }^{38}$ Yet, the major regulatory factors of inflammation-induced neurodegeneration in the SNpc a

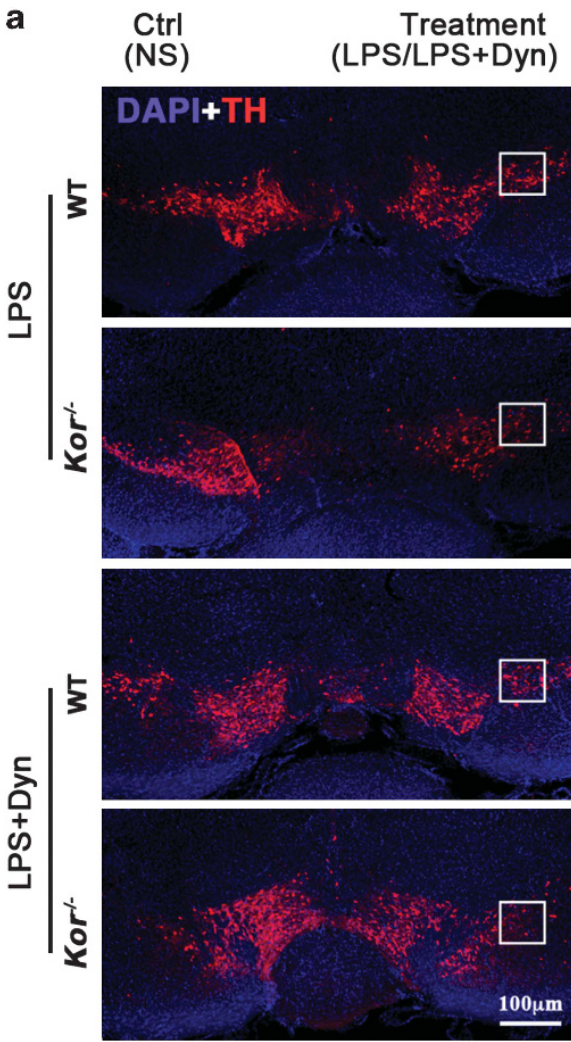

enlarged
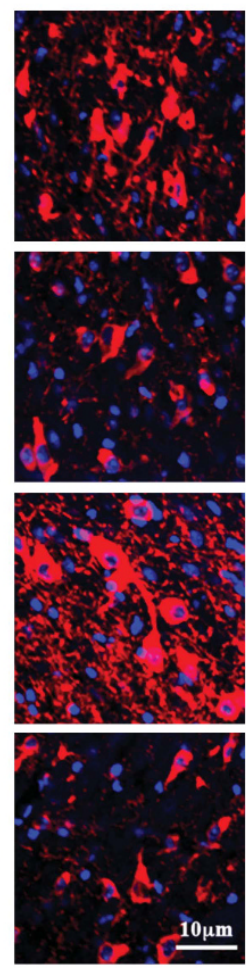

e

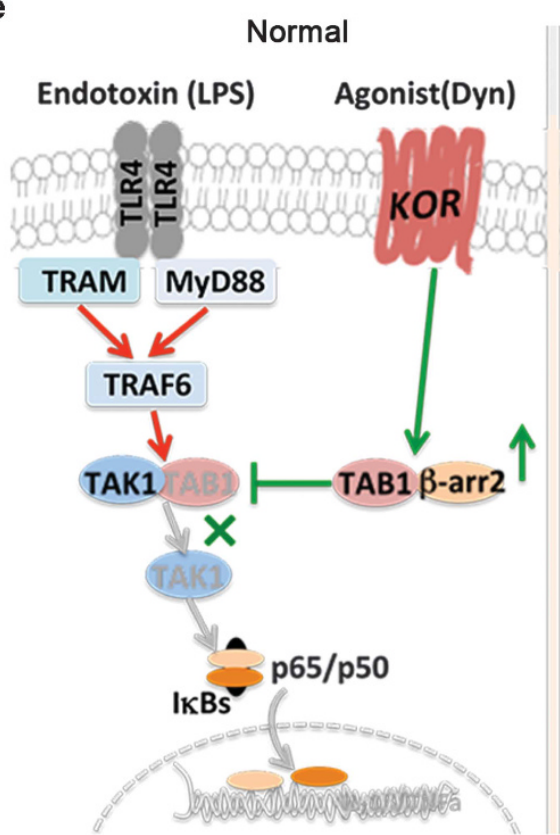

b
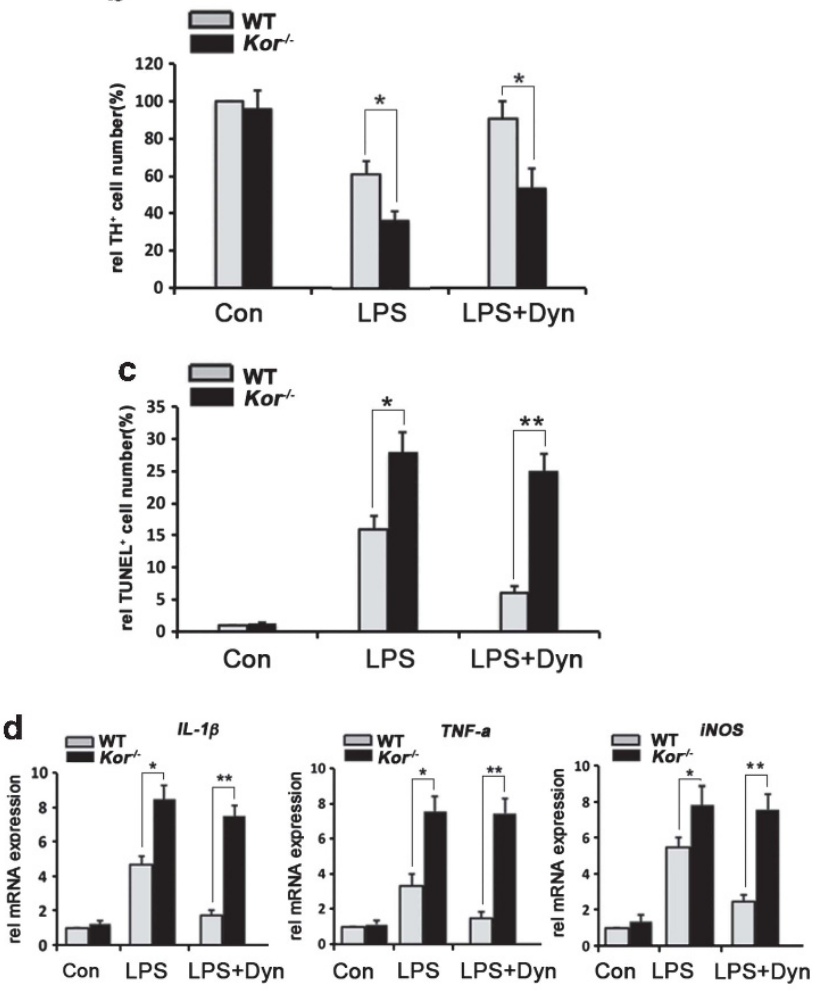

$\mathrm{KOR} / \beta$-arr2 KD

Endotoxin (LPS) Agonist(Dyn)

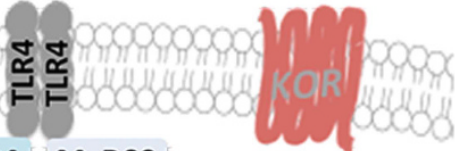

TRAM

MyD88

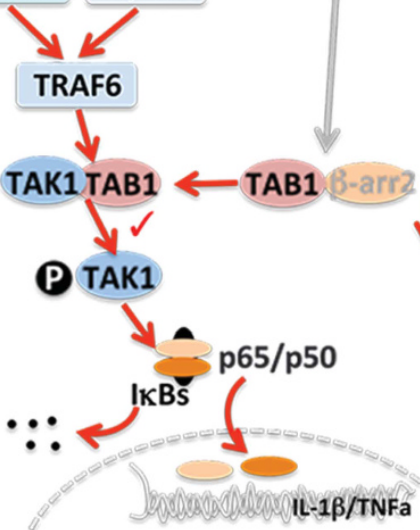


remained unknown. In the present study, significant DA neuronal loss was detected after injections of LPS into the $\mathrm{SN}$ of mice, whereas coadministration of Dyn, the endogenous agonist of KOR, reduced the number of LPSinduced neuronal deaths. However, this protective function of Dyn on neurons in response to LPS-induced inflammation was abolished in $\mathrm{Kor}^{-/-}$mice, demonstrating that the Dyn/KOR system is required to protect SN DA neurons from inflammation-induced neurotoxicity. We further show that the Dyn/KOR system that exerts this neuroprotective effect is an immune-derived microglial - not neuronal system, which acts through $\beta$-arrestin 'arrest' of these microglial cells' pro-inflammatory signaling and cytokine production.

Several other lines of evidence are consistent with our finding that KOR's neuroprotective role in the $\mathrm{SN}$ is its function as an inhibitor of pro-inflammatory gene expression in microglia, the brain macrophage. First, the neuroinflammatory response is predominately mediated by microglia, ${ }^{39}$ and limitation of pro-inflammatory and cytotoxic microglia through negative regulation of $\mathrm{NF}-\kappa \mathrm{B}$ activity protect $\mathrm{DA}$ neurons in chronic neuroinflammatory conditions. ${ }^{40}$ LPS is not effectively sensed by neurons. Second, as SN DA neurons are especially vulnerable to inflammatory insult, presumably because of the higher density of microglia in the $\mathrm{SN}$ than in other regions, ${ }^{41}$ intracerebral injections of LPS into the SN of mice have proven a well-established animal model to study acute inflammation-induced DA neurodegeneration. $^{29,42}$ Supporting these contentions, we detected significant neuronal death only in the neurons cocultured with LPS-treated microglia instead of in the neurons directly treated with LPS (Supplementary Figure S1b). Neuronal death was further enhanced in neurons cultured with the CM from LPS-treated microglia (or from LPS-treated microglia plus astrocytes) derived from $\mathrm{Kor}^{-/-}$mice. Although the activation of astrocytes by reactive microglia could be involved in the regulation of the inflammatory process, no significant neuronal death was detected from neurons cultured with CM from LPS-treated astrocytes alone, with or without KOR (Figure 1b). These results show that KOR protects neurons in response to inflammation derived from microglia.

In neurons, the Dyn/KOR system's effect on neurotransmitter release and behavioral changes has been well studied. ${ }^{43}$ KOR activation in DA neurons has been reported to inhibit the activity of the DA system, which has a critical role in the regulation of the brain reward system. ${ }^{44}$ However, how KOR functions in glial cells is still poorly understood. In this study, treating primary microglia with Dyn inhibited the LPS-induced increase of their inflammatory mediators IL-1 $\beta$, TNF- $\alpha$, and iNOS, whereas in $\mathrm{Kor}^{-/-}$microglia or mouse microglial cell lines (BV2 cells) with KOR knocked down by siRNA, the anti-inflammatory results of Dyn treatment were abolished (Figure 2). Similar to our microglial data, an exogenous KOR ligand, $\mathrm{U} 50,488 \mathrm{H}$, has been reported to inhibit the production of pro-inflammatory cytokines from primary murine macrophages. ${ }^{23}$ Our present study also confirms that exogenous KOR ligands (U50,488H, U60,593, and salvinorin A) similarly confer an anti-inflammatory effect (Supplementary Figure S2a). These results suggest that the anti-inflammatory role of Dyn is dependent on the KORmediated microglial signal pathway. This was further supported by our finding that Dyn inhibition of LPS-induced upregulation of pro-inflammatory cytokines, abolished in $\mathrm{Kor}^{-/-}$microglia, was rescued by transfecting them with KOR-IRES-GFP vector (Figures $2 d$ and e).

Countering brain inflammation largely mediated by immune cell-derived microglia is a therapeutic goal to retard the progression of neurodegenerative disorders like PD. Thus, it is important to mechanistically explore those signals that counter neurotoxic factors to develop such therapeutic interventions. LPS, as an endotoxin, is the ligand of TLR4, which has a central role in host cell recognition and immune responses to microbial pathogens by regulating pro-inflammatory gene expression through recruiting and enhancing TIRAP- and MyD88-dependent signaling pathways. ${ }^{35,36}$ Our results show that activation of microglial $\beta$-arrestin2, such as by Dyn/KOR activation, blocks LPS-induced phosphorylation of TAK1. Not only is $\beta$-arrestin2 a critical adaptor and regulator of internalization and desensitization of GPCRs like KOR, but it can also serve as a signal effector itself, having been previously reported to stabilize IkB by interacting with it, and as a result, to inhibit NF- $\kappa$ B activation in HEK293 cells. ${ }^{45}$ The expression of $\beta$-arrestin2 was previously reported also necessary to mediate the anti-inflammation function of $\beta 2$ adrenergic receptor in response to agonist stimulation. ${ }^{46}$ These suggest diverse role of $\beta$-arrestin2 in the regulation of GPCR signaling transduction. Thus, $\beta$-arrestin2 may represent a new target for anti-inflammatory therapy. However, the precise mechanism by which $\beta$-arrestin2 mediates GPCRrelated anti-inflammation role is still poorly understood. Our present study found that, in microglia, $\beta$-arrestin2 is normally associated with TAB1 and that this association is disrupted by pro-inflammatory TLR4 activation. This indicates diverse effects of $\beta$-arrestin2 on the TLR pathway in different cell types. Interestingly, we found that Dyn/KOR activation stabilizes $\beta$-arrestin2's association with TAB1. This competitively blocks TAB1's interaction with TAK1 and TAK1's subsequent phosphorylation, which attenuates the TLR4dependent signaling pathway (Figure $5 e$ ). Worthwhile, future studies could include investigating how microglial KOR or GPCR activation improves the translation efficiency of $\beta$-arrestin2 and stabilizes its sequestration of TAB1, but the present discovery of this novel microglial immuno-neuroprotective pathway identifies new clinical targets for therapeutic intervention of PD and other inflammation-dependent neurodegenerative diseases.

\section{Materials and Methods}

Animals and stereotaxic injection of LPS. All the animal experiments have been approved by the IACUC committee of the University of Minnesota and conform to the regulatory standards. The KOR-deficient $\left(\mathrm{Kor}^{-/-}\right)$mice was reported previously. ${ }^{47}$ Male adult (8-9 weeks old) or pregnant C57BL/6 mice from Charles River Laboratories (Chicago, IL, USA) were maintained and experimental procedures were conducted according to the $\mathrm{NIH}$ guidelines and approved by the University of Minnesota Institutional Animal Care and Use Committee (Protocol number 1007A86332). To induce inflammation-induced DA neuron death, LPS $\left(10 \mu \mathrm{g}\right.$ per animal, equal to $1 \times 10^{5} \mathrm{EU}$ endotoxin per animal) and LPS + Dyn ( $5 \mathrm{nmol}, 1 \mu \mathrm{l}$ in normal saline) were delivered to the right SN (bregma, $3.3 \mathrm{~mm}$; lateral, $1.2 \mathrm{~mm}$; vertical, $4.6 \mathrm{~mm}$ ) using a stereotaxic injection apparatus. ${ }^{48}$ The same volume of NS was injected to the left SN as control. 
Cell cultures. Primary DA neurons were isolated as previously described. ${ }^{49}$ Briefly, the SN tissues of embryo (E16-18) from WT or $\mathrm{Kor}^{-1-}$ mice were dissociated with $0.25 \%$ trypsin and cultured on poly-d-lysine-coated coverslips or Transwell polyester membrane inserts (3450, Corning, Tewksbury, MA, USA) in 12-well plates with neurobasal medium supplied with B27 and NGF. The plated cell density was $1.2 \times 10^{5}$ per well. The experiments were carried out after 12 days of culture (DIV 12). Primary microglia and astrocytes were separated from the brain of PO pups. After day 14 of glial mixture culture, microglia were prepared by mild trypsinization as previously described ${ }^{50}$ and maintained in DMEM-F12 medium supplemented with mouse M-CSF $(5 \mathrm{ng} / \mathrm{ml}), 10 \%$ FBS and $1 \%$ penicillin/ streptomycin. Primary astrocytes were separated from the cultured glial mixture by shaking the flasks in the culture box for $15 \mathrm{~h}$ at 250 r.p.m. and maintained in DMEM-F12 medium supplemented with 10\% FBS and 1\% penicillin/streptomycin. Murine microglial BV2 cells were maintained with DMEM supplemented with $10 \%$ FBS and 1\% penicillin/streptomycin. To activate TLR4-dependent inflammatory response, the cells were treated with $10 \mathrm{ng} / \mathrm{ml}$ LPS(100 EU endotoxin per $\mathrm{ml}$ ) for $2 \mathrm{~h}$.

CM treatment. Primary mouse microglia, astrocytes, or microglia-astrocyte mixtures were cultured in DMEM-F12 and washed with DMEM medium before treatment. The cells were then treated with $10 \mathrm{ng} / \mathrm{ml}(100 \mathrm{EU}$ endotoxin per $\mathrm{ml})$ LPS for $2 \mathrm{~h}$ and washed with DMEM extensively to avoid carryover of LPS to the next step. The cells were cultured in DMEM medium (supplied with $100 \mathrm{U} / \mathrm{ml}$ penicillin, 10\% FBS) for an additional $14 \mathrm{~h}$ and then the medium was collected as CM. CM was filtered through $0.45 \mu \mathrm{m}$ filters to remove dead cells. Neurons from $\mathrm{SN}$ of $\mathrm{Kor}^{-1-}$ mice or WT mice were cultured with CM (supplied with $500 \mathrm{ng} / \mathrm{ml}$ insulin, $0.02 \%$ sodium pyruvate and $2 \mathrm{mM}$ L-glutamine) for $24 \mathrm{~h}$, after which the neurons' survival was tested. The neuron cultured with DMEM was normal control.

Plasmid, siRNAs and cell transfection. The target sequences of siRNA against KOR or $\beta$-arrestin2 were: $5^{\prime}$-GAGCACCAATAGAGTTAGAAA- $3^{\prime}$ and $5^{\prime}-\mathrm{C}$ TGGCATCATCTGTTGGTATA-3' for KOR (NM 011011); $5^{\prime}$-CGGGACCAGGGTC TTCAAGAA-3', $5^{\prime}$-CCCGTGGCTCA-GCTAGAACAA- $3^{\prime}$ and $5^{\prime}$-CGGCTTATCATC AGAAAGGTA-3' for $\beta$-arrestin2 (NM_145429). KOR-IRES-GFP vector was made by inserting KOR CDNA into the Notl/Spel site of pShuttle-IRES-hrGFP-1 plasmid vector (Stratagene, Cambridge, MA, USA). Plasmid transfection was performed with Lipofectamine 2000 (Invitrogen, Carlsbad, CA, USA), and siRNA transfection was performed with Hyperfect reagent (Qiagen, Valencia, CA, USA).

IF and western blotting. Animals were anesthetized and perfused transcardially with $0.9 \%$ saline followed by $4 \%$ paraformaldehyde. Brain samples were post fixed with $4 \%$ paraformaldehyde overnight and equilibrated in 20 and $30 \%$ sucrose. Coronal sections of $40 \mu \mathrm{m}$ were prepared with a cryostat (CM1950, Leica, Allendale, NJ, USA).

Cells were fixed by $4 \%$ paraformaldehyde for $10 \mathrm{~min}$ and permeabilized using $0.2 \%$ TritonX-100 for $5 \mathrm{~min}$ at $4{ }^{\circ} \mathrm{C}$. Nonspecific binding was blocked by incubation of cells with $0.1 \%$ BSA-PBS $1 \mathrm{~h}$ before incubation with primary antibodies.

Immunofluorescence (IF) was performed using as primary antibody rabbit anti-TH IgG (Abcam, ab112, Cambridge, MA, USA) or rabbit anti-NF- $\kappa B$ P65 (Santa Cruz Biotechnology, sc-372, Dallas, TX, USA) and as secondary antibody Cy3-conjugated goat anti-mouse IgG (Santa Cruz Biotechnology). Nuclei were stained by DAPI (Sigma-Aldrich, St. Louis, MO, USA). Images were acquired with an Olympus FluoView 1000 IX2 upright confocal microscope (OLYMPUS, New York, NY, USA).

For western blotting, the cell lysates were prepared by sonication in RIPA buffer with protease and phosphatase inhibitor cocktails. Proteins $(60-80 \mu \mathrm{g})$ were resolved by $8 \%$ SDS-PAGE gel electrophoresis and transferred to PVDF membranes, blocked (in $5 \%$ milk) and incubated overnight in primary antibodies (anti-phospho-IKK, anti-IKK, anti-phospho-TAK1, and anti-TAK1 from Cell Signaling Technology, Beverly, MA, USA; anti-IkB, anti-p65, anti-laminA/C and anti- $\beta$-actin from Santa Cruz Biotechnology; anti-KOR, anti-TAB1, anti- $\beta$ - arrestin1 and anti$\beta$-arrestin2 from Abcam) followed by respective anti-IgG secondary antibodies. Membranes were developed for visualization and photography using ECL reagent (Thermo, Rockford, IL, USA).

Immunoprecipitation and in situ PLA. Immunoprecipitation and in situ PLA were performed as described previously ${ }^{51}$ using antibodies (anti-TAK1, anti-TAB1; anti- $\beta$-arrestin1 and anti- $\beta$-arrestin2 from Abcam) or Duolink PLA kit (Olink Bioscience, Uppsala, Sweden) after fixation of cells with $4 \%$ paraformaldehyde. Images were acquired with an Olympus FluoView 1000 IX2 upright confocal microscope. Fluorescent punctae representing protein complexes in PLA were counted using Image $\mathrm{J}$ image processing freeware (http://rsbweb.nih.gov/ij).

Gene expression assay by qPCR. Total RNA was isolated from microglial cells or the SN from the brain by TRIzol reagent (Invitrogen). RNA samples' cDNA was synthesized using Omniscript RT kit (Qiagen), and qPCR was performed with SYBR-Green QPCR reagent (Agilent, Santa Clara, CA, USA) and detected by the Mx3005P QPCR system (Agilent). qPCR primer sequences used for mRNA quantification were the following: forward $5^{\prime}$-TCAGGCAGGCAGTATCA CTCA-3', reverse $5^{\prime}$-GGAAGGTCCACGGGAAAGAC- $3^{\prime}$ for IL- $1 \beta$; forward $5^{\prime}$-ATG AGCACAGAAAGCATGATCCGC- $3^{\prime}$, reverse $5^{\prime}$-CCAAAGTAGACCTGCCCGGAC TC- $3^{\prime}$ for TNF- $\alpha$; forward $5^{\prime}$ - ACCCACATCTGGCAGAATGAG-3', reverse $5^{\prime}$ - AGC CATGACCTTTCGCATTAG-3' for iNOS; forward $5^{\prime}$-GCAGCCTGAATCCTGTT CTC- $3^{\prime}$, reverse $5^{\prime}$-TCATCCCTCCCACATCTCTC-3' for KOR and forward $5^{\prime}$-GG CAAGCGCGACTTTGTAG- $3^{\prime}$, reverse $5^{\prime}$-GTGAGGGTCACGAACACTTTC-3' for $\beta$-arrestin2.

Measurement of cell survival and apoptosis. The cell survival was indicated by spectrophotometric measurement of cell viability using MTT-based In Vitro Toxicology Assay Kit (TOX1-1KT, Sigma-Aldrich) according to the instruction of protocol. Absorbance was measured at $544 \mathrm{~nm}$ using FLUOstar GalaxyMultidetection Microplate Reader (BMG Labtech, Cary, NC, USA). The results representing cell survival was analyzed to the percentage of control group.

Cell apoptosis was detected using the CytoGLO SIVA-IANBD kit (IMG-6701K, IMGENEX, San Diego, CA, USA) and CF594 TUNEL apoptosis detection kit (30064, Biotium, Hayward, CA, USA) according to their protocols. Images for pSIVA, PI and TUNEL were acquired with an Olympus FluoView 1000 IX2 upright confocal microscope. The fluorescence-positive cells representing apoptotic cells were quantified using Image $\mathrm{J}$.

Stereological analysis of $\mathrm{TH}^{+}$neurons in the SNpc. Systematic random sampling and unbiased stereological estimates were performed for quantification of $\mathrm{TH}^{+}$neurons and apoptotic cells as previously described. ${ }^{52,53}$ For each animal, a total of $40(40 \mu \mathrm{m})$ sections containing the entire SN (between -2.40 and $-4.20 \mathrm{~mm}$ from the bregma) were cut and five of the sections were sampled. The first section sampled was selected randomly and the sampling interval thereafter was determined based on the number of sections within the SN and was usually every eighth section. For each selected section, a lowmagnification $(\times 2)$ image was captured. A grid was randomly cast over the image and systematic random sampling was again used when choosing the grid intersections to be evaluated. Once an intersection was chosen, a stack of images was made at $\times 40$ while focusing through the full thickness of the section.

Cells were counted as follows: a counting frame was superimposed on the stack of images. The right boundary and the upper boundary of the counting frame were used as acceptance lines; the other two boundaries were forbidden lines. The THpositive neurons were counted only if NeuN-labeled nuclei and $\mathrm{TH}$-immunoreactive profiles fell entirely inside the counting frame or if they crossed an acceptance line without also crossing a forbidden line. NeuN-labeled neurons were counted when the top of the nucleus was found within the thickness of the tissue section.

To estimate the total number of $\mathrm{TH}^{+}$neurons $(M)$ in the SN per animal, we used the following equation: $N=(\Sigma(D \times V)) / n$. Where $D$ is the neuronal density of the $S N$ and $V$ is the volume of the $\mathrm{SN}$.

To estimate the neuronal density of the SN in each animal, we used the following equation: $D=N /(a \times t \times g)$. Where $N^{\prime}$ is the number of neurons counted per animal, $a$ is the area of the counting grids sampled, $t=40 \mu \mathrm{m}$ (the thickness of the section sampled), and $g=20$ (the number of counting frames sampled per animal).

To estimate the volume of the SN from each animal, we used the following equation: $V=\Sigma(A \times I \times t)$. Where $A$ is the cross-sectional area of SN in which the sections were sampled, $I$ is the interval between adjacent sampled sections, and $t=40 \mu \mathrm{m}$ (the thickness of section sampled).

Statistical analyses. Statistical significance for multiple comparisons was determined by Student's t-test using SPSS17.0 software (SPSS, Quarry Bay, Hong Kong) and summarized as the mean \pm S.D. of repeated measures; $P<0.05$ was considered statistically significant at the $95 \%$ level.

\section{Conflict of Interest}

The authors declare no conflict of interest. 
Acknowledgements. We thank Martin Wessendorf, Y-W Lin, A Smith, A Kim and I Zhang for technical help. This work was supported by NIH Grants DK54733, DK60521, DA11190, DA01583, and DA11806, the Dean's Commitment (LNW), and the Distinguished McKnight Professorship of University of Minnesota (LNW).

1. Imamura K, Hishikawa N, Sawada M, Nagatsu T, Yoshida M, Hashizume Y. Distribution of major histocompatibility complex class II-positive microglia and cytokine profile of Parkinson's disease brains. Acta Neuropathol 2003; 106: 518-526.

2. Kim W-G, Mohney RP, Wilson B, Jeohn GH, Liu B, Hong JS. Regional difference in susceptibility to lipopolysaccharide-induced neurotoxicity in the rat brain: role of microglia. J Neurosci 2000; 20: 6309-6316.

3. McGeer PL, Itagaki S, Boyes BE, McGeer EG. Reactive microglia are positive for HLA-DR in the substantia nigra of Parkinson's and Alzheimer's disease brains. Neurology 1988; $\mathbf{3 8}$ : 1285-1291.

4. Block ML, Zecca L, Hong J-S. Microglia-mediated neurotoxicity: uncovering the molecular mechanisms. Nat Rev Neurosci 2007; 8: 57-69.

5. Biber K, Neumann H, Inoue K, Boddeke HWGM. Neuronal on and off signals control microglia. Trends Neurosci 2007; 30: 596-602.

6. Harms AS, Barnum CJ, Ruhn KA, Varghese S, Treviño I, Blesch A et al. Delayed dominant-negative tnf gene therapy halts progressive loss of nigral dopaminergic neurons in a rat model of Parkinson's disease. Mol Ther 2011; 19: 46-52.

7. McCoy MK, Martinez TN, Ruhn KA, Szymkowski DE, Smith CG, Botterman BR et al. Blocking soluble tumor necrosis factor signaling with dominant-negative tumor necrosis factor inhibitor attenuates loss of dopaminergic neurons in models of Parkinson's disease. J Neurosci 2006; 26: 9365-9375.

8. Zhang F, Qian L, Flood PM, Shi JS, Hong JS, Gao HM. Inhibition of IкB kinase- $\beta$ protects dopamine neurons against lipopolysaccharide-induced neurotoxicity. J Pharmacol Exp Ther 2010; 333: 822-833.

9. Block ML, Hong JS. Chronic microglial activation and progressive dopaminergic neurotoxicity. Biochem Soc Trans 2007; 35: 1127-1132.

10. Kim YS, Choi DH, Block ML, Lorenzl S, Yang L, Kim YJ et al. A pivotal role of matrix metalloproteinase-3 activity in dopaminergic neuronal degeneration via microglial activation. FASEB J 2007; 21: 179-187.

11. Meredith G, Sonsalla P, Chesselet M-F. Animal models of Parkinson's disease progression. Acta Neuropathol 2008; 115: 385-398.

12. Chavkin C, James I, Goldstein A. Dynorphin is a specific endogenous ligand of the kappa opioid receptor. Science 1982; 215: 413-415.

13. DeWire SM, Ahn S, Lefkowitz RJ, Shenoy SK. Beta-arrestins and cell signaling. Ann Rev Physiol 2007; 69: 483-510.

14. Bruijnzeel AW. Kappa-opioid receptor signaling and brain reward function. Brain Res Rev 2009; 62: 127-146.

15. Ikeda K, Yoshikawa S, Kurokawa T, Yuzawa N, Nakao K, Mochizuki H. TRK-820, a selective kappa opioid receptor agonist, could effectively ameliorate L-DOPA-induced dyskinesia symptoms in a rat model of Parkinson's disease. Eur J Pharmacol 2009; 620: 42-48.

16. Manzanares J, Lookingland KJ, Moore KE. Kappa opioid receptor-mediated regulation of dopaminergic neurons in the rat brain. J Pharmacol Exp Ther 1991; 256: 500-505.

17. Margolis EB, Hjelmstad GO, Bonci A, Fields HL. $\kappa$-Opioid agonists directly inhibit midbrain dopaminergic neurons. J Neurosc 2003; 23: 9981-9986.

18. Waters CM, Peck R, Rossor M, Reynolds GP, Hunt SP. Immunocytochemical studies on the basal ganglia and substantia nigra in Parkinson's disease and Huntington's chorea. Neuroscience 1988; 25: 419-438.

19. Carta A, Fenu S, Morelli M. Alterations in GAD67, dynorphin and enkephalin mRNA in striatal output neurons following priming in the 6-OHDA model of Parkinson's disease. Neurol Sci 2001; 22: 59-60.

20. Wang Q, Shin EJ, Nguyen XK, Li Q, Bach JH, Bing G et al. Endogenous dynorphin protects against neurotoxin-elicited nigrostriatal dopaminergic neuron damage and motor deficits in mice. J Neuroinflammation 2012; 9: 124.

21. Bidlack JM. Detection and function of opioid receptors on cells from the immune system. Clin Diagn Lab Immunol 2000; 7: 719-723.

22. Alicea C, Belkowski SM, Sliker JK, Zhu J, Liu-Chen LY, Eisenstein TK et al. Characterization of kappa-opioid receptor transcripts expressed by $\mathrm{T}$ cells and macrophages. J Neuroimmunol 1998; 91: 55-62.

23. Alicea C, Belkowski S, Eisenstein TK, Adler MW, Rogers TJ. Inhibition of primary murine macrophage cytokine production in vitro following treatment with the K-opioid agonist U50, 488H. J Neuroimmunol 1996; 64: 83-90.

24. Belkowski SM, Alicea C, Eisenstein TK, Adler MW, Rogers TJ. Inhibition of interleukin-1 and tumor necrosis factor-alpha synthesis following treatment of macrophages with the kappa opioid agonist U50, 488H. J Pharmacol Exp Ther 1995; 273: 1491-1496.
25. Chao CC, Gekker G, Hu S, Sheng WS, Shark KB, Bu DF et al. kappa opioid receptors in human microglia downregulate human immunodeficiency virus 1 expression. Proc Natl Acad Sci 1996; 93: 8051-8056.

26. Chao CC, Hu S, Gekker G, Lokensgard JR, Heyes MP, Peterson PK. U50,488 protection against HIV-1-related neurotoxicity: involvement of quinolinic acid suppression. Neuropharmacology 2000; 39: 150-160.

27. Hu S, Peterson PK, Chao CC. $\kappa$-Opioid modulation of human microglial cell superoxide anion generation. Biochem Pharmacol 1998; 56: 285-288.

28. Wee S, Koob GF. The role of the dynorphin-kappa opioid system in the reinforcing effects of drugs of abuse. Psychopharmacology 2010; 210: 121-135.

29. Saijo K, Winner B, Carson CT, Collier JG, Boyer L, Rosenfeld MG et al. A Nurr1/CoREST pathway in microglia and astrocytes protects dopaminergic neurons from inflammationinduced death. Cell 2009; 137: 47-59.

30. Bhaskar K, Konerth M, Kokiko-Cochran ON, Cardona A, Ransohoff RM, Lamb BT. Regulation of tau pathology by the microglial fractalkine receptor. Neuron 2010; 68: 19-31.

31. Luttrell LM, Lefkowitz RJ. The role of beta-arrestins in the termination and transduction of G-protein-coupled receptor signals. J Cell Sci 2002; 115: 455-465.

32. Zheng H, Loh HH, Law PY. Beta-arrestin-dependent mu-opioid receptor-activated extracellular signal-regulated kinases (ERKs) Translocate to nucleus in contrast to $G$ protein-dependent ERK activation. Mol Pharmacol 2008; 73: 178-190.

33. Loniewski K, Shi Y, Pestka J, Parameswaran N. Toll-like receptors differentially regulate GPCR kinases and arrestins in primary macrophages. Mol Immunol 2008; 45: 2312-2322.

34. Fan H, Luttrell LM, Tempel GE, Senn JJ, Halushka PV, Cook JA. Beta-arrestins 1 and 2 differentially regulate LPS-induced signaling and pro-inflammatory gene expression. $\mathrm{Mol}$ Immunol 2007; 44: 3092-3099.

35. Kawai T, Akira S. Toll-like receptors and their crosstalk with other innate receptors in infection and immunity. Immunity 2011; 34: 637-650.

36. Kawai T, Akira S. TLR signaling. Cell Death Differ 2006; 13: 816-825.

37. Hirsch EC, Hunot S. Neuroinflammation in Parkinson's disease: a target for neuroprotection? Lancet Neurol 2009; 8: 382-397.

38. Gao HM, Liu B, Zhang W, Hong JS. Novel anti-inflammatory therapy for Parkinson's disease. Trends Pharmacol Sci 2003; 24: 395-401.

39. Lawson LJ, Perry VH, Dri P, Gordon S. Heterogeneity in the distribution and morphology of microglia in the normal adult mouse brain. Neuroscience 1990; 39: 151-170.

40. Lee J-K, Chung J, McAlpine FE, Tansey MG. Regulator of G-protein signaling-10 negatively regulates NF-kB in microglia and neuroprotects dopaminergic neurons in Hemiparkinsonian rats. J Neurosci 2011; 31: 11879-11888.

41. Kim WG, Mohney RP, Wilson B, Jeohn GH, Liu B, Hong JS. Regional difference in susceptibility to lipopolysaccharide-induced neurotoxicity in the rat brain: role of microglia. J Neurosci 2000; 20: 6309-6316.

42. Meredith GE, Sonsalla PK, Chesselet MF. Animal models of Parkinson's disease progression. Acta Neuropathol 2008; 115: 385-398.

43. Flaisher-Grinberg S, Persaud SD, Loh HH, Wei LN. Stress-induced epigenetic regulation of kappa-opioid receptor gene involves transcription factor c-Myc. Proc Natl Acad Sci USA 2012; 109: 9167-9172.

44. Bruijnzeel AW. Kappa-opioid receptor signaling and brain reward function. Brain Res Rev 2009; 62: 127-146.

45. Gao H, Sun Y, Wu Y, Luan B, Wang Y, Qu B et al. Identification of beta-arrestin2 as a G protein-coupled receptor-stimulated regulator of NF-kappaB pathways. Mol Cell 2004; 14: 303-317.

46. Qian L, Wu HM, Chen SH, Zhang D, Ali SF, Peterson L et al. beta2-adrenergic receptor activation prevents rodent dopaminergic neurotoxicity by inhibiting microglia via a novel signaling pathway. J Immunol 2011; 186: 4443-4454.

47. Simonin F, Valverde O, Smadja C, Slowe S, Kitchen I, Dierich A et al. Disruption of the [kappa]-opioid receptor gene in mice enhances sensitivity to chemical visceral pain, impairs pharmacological actions of the selective [kappa]-agonist $\mathrm{U}-50,488 \mathrm{H}$ and attenuates morphine withdrawal. EMBO J 1998; 17: 886-897.

48. Paxinos G, Franklin KBJ. The Mouse Brain In Stereotaxic Coordinates. 2nd edn, Elsevier Academic Press: Amsterdam; Boston, 2004.

49. Fath T, Ke YD, Gunning P, Gotz J, Ittner LM. Primary support cultures of hippocampal and substantia nigra neurons. Nat Protoc 2009; 4: 78-85.

50. Saura J, Tusell JM, Serratosa J. High-yield isolation of murine microglia by mild trypsinization. Glia 2003; 44: 183-189.

51. Ho PC, Tsui YC, Feng X, Greaves DR, Wei LN. NF-kappaB-mediated degradation of the coactivator RIP140 regulates inflammatory responses and contributes to endotoxin tolerance. Nat Immunol 2012; 13: 379-386.

52. Leong ML, Gu M, Speltz-Paiz R, Stahura El, Mottey N, Steer CJ et al. Neuronal loss in the rostral ventromedial medulla in a rat model of neuropathic pain. $J$ Neurosci $2011 ; 31$ : 17028-17039.

53. Howard CV, Reed MG. Unbiased Stereology: Three-Dimensional Measurement In Microscopy. BIOS Scientific: Oxford, UK, 1998 